\title{
REVISIÓN
}

\section{Actividad antiviral de compuestos aislados de esponjas marinas}

\author{
Antiviral activity of compounds isolated from marine sponges
}

\section{León G. Gómez-Archila ${ }^{1}$, María T. Rugeles ${ }^{1}$ y Wildeman Zapata ${ }^{1,2}$}

\begin{abstract}
${ }^{1}$ Grupo Inmunovirología, Facultad de Medicina, Universidad de Antioquia, código postal 050034, Medellín, Colombia ${ }^{2}$ Grupo Infettare, Facultad de Medicina, Universidad Cooperativa de Colombia, código postal 050034, Medellín, Colombia. wildeman.zapatab@campusucc.edu.co
\end{abstract}

\begin{abstract}
Marine sponges have been used for the isolation and purification of compounds with therapeutic properties for human use. These compounds are used mainly against viruses because these microorganisms mutate and create resistance easily to available treatments. In the last 60 years, marine sponges have been the subject of scientific research, which have addressed the discovery of compounds with activity against Herpes Simplex Virus (HSV) such as Ara-A; the avarol used against Human Immunodeficiency Virus Type 1 (HIV-1) and calyceramides against Influenza Virus. This article present a review of the chemistry and mechanism of action of the compounds isolated from marine sponges that have shown antiviral activity, to encourage the search for new molecules or their modification in order to obtain several sources of drug production and antiviral treatments. This review found that in vitro cell models have been the most used techniques and the HSV and HIV-1 are the main microorganisms studied for the determination of the antiviral activity; finally, it was found that the biological activities are directly related to the structure of the compounds, especially when they are analogs of amino acids or nucleotides.
\end{abstract}

Key words: Marine sponge, antiviral activity, Herpes simplex virus (HSV), Human Immunodeficiency Virus Type 1 (HIV-1)

Resumen.- Las esponjas marinas se han utilizado para el aislamiento y purificación de compuestos con propiedades terapéuticas para uso en humanos, los cuales se usan principalmente contra virus, debido a que estos microorganismos mutan y crean fácilmente resistencia a los tratamientos disponibles. En los últimos 60 años las esponjas marinas han sido objeto de investigaciones científicas, las cuales han permitido el descubrimiento de compuestos como el Ara-A, avarol y las caliceramidas con actividad contra el Virus Herpes Simple (VHS), el Virus de la Inmunodeficiencia Humana tipo 1(VIH1) y el Virus de Influenza, respectivamente. Este trabajo presenta una revisión de la química y el mecanismo de acción de los compuestos aislados de esponjas marinas que han mostrado actividad antiviral, de modo que se incentive la búsqueda de nuevas moléculas o su modificación con el fin de conseguir fuentes de producción de medicamentos y tratamientos antivirales. La revisión plantea que los modelos celulares in vitro han sido las técnicas más utilizadas y que el VHS y el VIH1 son los microorganismos más estudiados para la determinación de la actividad antiviral; finalmente, se sugiere que las actividades biológicas en general están directamente relacionadas con la estructura de los compuestos, en especial cuando son análogos de aminoácidos o nucleótidos.

Palabras clave: Esponjas marinas, actividad antiviral, Virus Herpes Simple (VHS), Virus de la Inmunodeficiencia Humana tipo $1(\mathrm{VIH}-1)$

\section{INTRODUCCIÓN}

El lecho marino es una gran fuente de recursos naturales y constituye para el hombre un campo de alto interés para el aprovechamiento sostenible de la biodiversidad. En el caso de Colombia, por tener una posición geográfica privilegiada y costas tropicales sobre los Océanos Atlántico y Pacífico, las cuales representan el 50\% del territorio nacional, se cuenta con una gran diversidad biótica marina de aproximadamente 90.000 especies (entre algas, esponjas, corales, anélidos, moluscos, crustáceos y peces) (Zea 1998) y se estima una población de más de
1.000 especies de esponjas arrecifales. A pesar de esto, los estudios actuales no llegan a cubrir el $10 \%$ del inventario marino, desconociendo el potencial de este recurso como fuente de nuevas alternativas terapéuticas.

El interés farmacéutico por las esponjas surgió en la década de 1950 con el descubrimiento de los nucleósidos espongotimidina y espongouridina en la esponja marina Cryptotethia cripta (Bergmann \& Feeney 1950, 1951). Estos nucleósidos fueron la base para la síntesis de Ara-C, el primer agente de origen marino contra la 
leucemia mieloide aguda y el linfoma no Hodgkin y del fármaco antiviral Ara-A usado contra el Virus Herpes Simple (VHS) (Privat de Garilhe 1964); uno de sus derivados fluorados también ha sido aprobado para el uso en pacientes con cáncer de páncreas, mama, vejiga y pulmón (Sipkema et al. 2005).

La diversidad de los metabolitos secundarios aislados de esponjas es muy amplia; se han descrito más de 15.000 sustancias, que son responsables de más de 5.300 productos diferentes entre los que se pueden encontrar terpenos, esteroles, nucleósidos inusuales, péptidos cíclicos, alcaloides, ácidos grasos, peróxidos y derivados de aminoácidos (con frecuencia halogenados) (Tziveleka et al. 2003, Sipkema et al. 2005). La aparición de especies marinas desde inicios de la cadena evolutiva les ha otorgado suficiente tiempo para desarrollar un sistema de defensa químico avanzado en respuesta a estímulos particulares, con el fin de protegerse de microorganismos y depredadores; por esta razón se han convertido en materia de estudio en la búsqueda de compuestos antimicrobianos en los últimos años (Sipkema et al. 2005).

El creciente interés en los compuestos antivirales de origen marino, junto con el desarrollo de nuevas tecnologías en cultivos marinos y en los procesos de extracción de distintas moléculas, han acelerado de manera significativa la exploración del medio marino como fuente significativa de compuestos con aplicaciones farmacológicas importantes (Pérez-López et al. 2014). Aunque existentes limitantes en la producción de los compuestos y su posterior producción en cantidades industriales por medio de rutas biológicas o biotecnológicas (Gerwick \& Moore 2012).

Estos aspectos en conjunto, crean un escenario único e ideal para la búsqueda de sustancias bioactivas naturales de origen marino que pueden contribuir al tratamiento de distintas enfermedades, en particular aquellas de impacto mundial que afectan a los seres humanos.

En la presente revisión se abordó el tema de las esponjas marinas como fuente potencial de compuestos antivirales, realizando una breve descripción de este grupo marino y las características que describen su importancia como moléculas medicinales, a través de una revisión bibliográfica en bases de datos científicas.

\section{ESPONJAS MARINAS}

Entre las diversas formas de vida marina, las esponjas del Phylum Porífera, son animales invertebrados, pluricelulares simples y sésiles que solo tienen movimiento durante su estado larval hasta que se depositan sobre un sustrato; han demostrado ser el mayor reservorio de compuestos bioactivos novedosos en el lecho marino como alcaloides, esteroles, terpenoides, lípidos, macrólidos, péptidos cíclicos, esteres de ácidos grasos y poliéteres (Zhang et al. 2003).

Las esponjas marinas dominan en los ecosistemas marinos, hábitats crípticos y poco iluminados como cuevas y túneles, y en los corales al no competir con organismos fotosintéticos de crecimiento más rápido; en arrecifes coralinos por debajo de los $20 \mathrm{~m}$ también se observa un predominio, mientras que en canales más profundos como los manglares son las raíces adventicias (Rützler \& Feller 1987). Las esponjas juegan un papel importante en los ecosistemas marinos pues muchas especies albergan simbiontes fotosintéticos, especialmente cianobacterias y tienen numerosas bacterias heterotróficas en sus tejidos las cuales devuelven al medio nutrientes remineralizados (Corredor et al. 1988); además, constituyen el hábitat de muchos invertebrados y algunos peces.

La mayoría de estas especies presentan sistemas inmunológicos complejos, mediante los cuales producen compuestos químicos en respuesta a estímulos particulares, con el fin de protegerse contra hongos, bacterias, virus y depredadores (Narsinh \& Werner 2004). Esta respuesta da lugar a la síntesis de un amplio número de sustancias especializadas, denominadas metabolitos secundarios, muchos de los cuales exhiben efectos biológicos de alta selectividad y potencia para poder contrarrestar la dilución a la que tienen que hacer frente en el medio acuoso que les rodea, ofreciendo así una fuente de compuestos potencialmente útiles para el desarrollo de medicamentos (Kong \& Andersen 1996).

\section{Metabolitos SECUNDARIOS DE LAS ESPONJAS}

Los metabolitos primarios son compuestos orgánicos como azúcares, aminoácidos, ácidos grasos, nucleótidos y polímeros derivados como polisacáridos, lípidos, proteínas, ADN y ARN, que son necesarios para el desarrollo de los organismos vivos (Piñol \& Palazón 1993). Por otra parte, los metabolitos secundarios son subproductos de las rutas metabólicas primarias, siendo específicos de un grupo taxonómico y estado de vida o tejido. Con pocas excepciones, los metabolitos secundarios pueden ser clasificados dentro de 3 grupos, de acuerdo con su base biosintética: derivados fenólicos, derivados terpénicos y derivados nitrogenados. Su distribución depende de condiciones externas tales como 
retos por microorganismos patógenos, predadores, cambios térmicos o lumínicos, deficiencias nutricionales o presencia de otros organismos intra o interespecíficos (Bula-Meyer 1989). El metabolismo secundario es una característica fundamental de la especialización, es decir que el compuesto resultante, puede no ser importante para la célula pero sí para el organismo como un todo.

Las esponjas marinas como las plantas terrestres, producen diferentes metabolitos secundarios tales como: compuestos acetilénicos, alcaloides, esteroles, macrólidos, péptidos, peróxidos, terpenos, etc. A diferencia de las plantas, las esponjas incorporan halógenos a sus metabolitos secundarios y producen compuestos nitrogenados como alcaloides (Bickmeyer et al. 2004, Aiello et al. 2006).

Los metabolitos secundarios aislados de esponjas marinas muestran una alta variedad de actividades biológicas, entre las que se destacan, la actividad anticancerígena exhibida por la dragmacidina 1, aislada de la esponja que lleva el mismo nombre con una concentración inhibitoria $50\left(\mathrm{CI}_{50}\right)$ de $15 \mu \mathrm{g} \mathrm{mL}^{-1}$ en la línea celular P-388 de leucemia murina y entre 1 y $10 \mu \mathrm{g}$ $\mathrm{ml}^{-1}$ contra las líneas celulares A-549 (cáncer de pulmón), HCT-8 (cáncer de colon), y MDAMB (cáncer de tejido mamario) (Gul \& Hamann 2005); la 11-Deoxifistularina-3, aislada de Aplysina fistularis insularis es citotóxica para la línea celular MCF-7 (cáncer de seno), con una dosis letal $50\left(\mathrm{DL}_{50}\right)$ de $17 \mu \mathrm{g} \mathrm{mL} \mathrm{m}^{-1}$ (Compagnone et al. 1999); la actividad anti-leishmania expuesta por una fracción de fluido supercrítico de Tabernaemontana catharinensis, inhibiendo la replicación de amastigotes de Leishmania amazonensis en macrófagos, con un porcentaje de inhibición de la supervivencia del parásito de 88, 41, y 36\% a concentraciones de 100,10 y $1 \mu \mathrm{g} \mathrm{mL}{ }^{-1}$ respectivamente (Soares et al. 2007); la actividad antienzimática expuesta por la Aplysamina 6, aislada de la esponja Pseudoceratina sp. la cual mostró actividad de inhibición de la isoprenilcisteína-carboxil-metil-transferasa (ICMT) con una $\mathrm{CI}_{50}$ de $14 \mu \mathrm{M}$ (Buchanan et al. 2008); la actividad hemolítica y anti-malárica mostrada por el 6-carbometoximetil-3-metoxi1,2-dioxano contra el Plasmodium falciparum con una $\mathrm{CI}_{50}$ de 0,12 $\mu \mathrm{M}$ (Murakamia et al. 2003), entre otras (Márquez et al. 2003, 2004; Galeano \& Martínez 2007).

Es importante resaltar que en el periodo entre 1988 y 2011 se reportó una alta variedad de especies de esponjas con un amplio rango de actividad antiviral debido principalmente a la implementación de los ensayos in vitro que permiten la obtención de resultados rápidos (Márquez et al. 2003, 2004). A continuación se hace un breve resumen de esta actividad y de los diferentes virus sobre los cuales se ha encontrado que actúan los compuestos aislados de esponjas marinas.

\section{ACTIVIDAd ANTIVIRal DE LAS ESPONJAS MARINaS}

Las esponjas marinas han sido consideradas en los últimos 60 años como una fuente importante de compuestos biológicamente activos debido a la diversidad de sus metabolitos secundarios. Más de 5.300 compuestos naturales se han descubierto en las esponjas y sus microorganismos asociados, y cientos de nuevos compuestos se agregan cada año (Faulkner 2002). Por lo tanto, las esponjas tienen el potencial de proporcionar futuros fármacos contra enfermedades importantes, tales como el cáncer, infecciones bacterianas, virales y parasitarias, así como en los procesos inflamatorios (Sipkema et al. 2005).

\section{VIRUS DEL HERPES SIMPLE (VHS)}

Varios autores han reportado compuestos aislados de esponjas marinas con actividad anti-VHS, y varios compuestos activos se han aislado y caracterizado (Tabla 1). Dos nucleósidos: la espongotimidina y la espongouridina fueron aislados de la esponja del Caribe Cryptotethya cripta (Bergmann \& Burke 1955). La espongotimidina se encontró selectiva contra el VHS tipo 1 (VHS-1) y tipo 2 (VHS-2) con una $\mathrm{CI}_{50}$ de 0,25 y $0,5 \mu \mathrm{g}$ $\mathrm{mL}^{-1}$, respectivamente (Aswell et al. 1977, Miller et al. 1977).

Por otro lado, la espongouridina se ha utilizado como una estructura química de molde para la síntesis del AraA y mejorar su actividad anti-VHS (Privat de Garilhe 1964, Schwartz et al.1976, Utagawa et al. 1980). Este se licenció como el primer nucleósido anti-VHS y uno de los 3 fármacos de origen marino actualmente aprobados por la Administración de Alimentos y Medicamentos de los Estados Unidos (FDA) (Mayer et al. 2010).

El Ara-A tiene una potente actividad antiviral contra el VHS-1 y 2; el mecanismo de acción se basa en que el AraA detiene la replicación del ADN viral de 3 formas: 1) inhibición competitiva de la ADN polimerasa viral, 2) incorporación y finalización del crecimiento de la cadena de ADN viral, y 3) la inactivación de la ADN polimerasa viral (Yasuhara-Bell \& Lu 2010). Al ser un análogo de nucleósido tiene que ser fosforilado para estar activo; este es un proceso que consta de 3 etapas en las que el 
Tabla 1. Compuestos aislados de esponjas marinas con actividad anti-VHS / Compounds isolated from marine sponges with anti-HSV activity

\begin{tabular}{|c|c|c|c|}
\hline Especie & Sustancia & Clase & Referencia \\
\hline Cryptotethya crypta & Espongotimidina y Ara-A & Nucleósido & Bergmann \& Burke (1955) \\
\hline Cryptotethya crypta & Ara-C & Nucleósido & Privat de Garilhe \& de Rudder (1964) \\
\hline Mycale sp. & Micalamida A-B & Nucleósido & Perry et al. $(1988,1990)$ \\
\hline Ptilocaulis spiculifer & $\begin{array}{l}\text { Guanidina policíclica } \\
\text { Ptilomicalia A }\end{array}$ & Alcaloide & Kashman et al. (1989) \\
\hline Aaptos aaptos & 4-Metilaptamina & Alcaloide & $\begin{array}{l}\text { Coutinho et al. (2002), Souza et } \\
\quad \text { al. }(2007)\end{array}$ \\
\hline Halicortex sp. & Dragmacidina F & Alcaloide & Cutignano et al. (2000) \\
\hline Pachypellina sp. & 8-Hidroximanzamina & Alcaloide & Ichiba et al. (1994) \\
\hline Hamigera tarangaensis & Hamigerana B & Macrólido fenólico & Wellington et al. (2000) \\
\hline Cliona sp. Agelas sp. y Tethya sp. & Extractos acuosos & & Da Silva et al. (2006) \\
\hline
\end{tabular}

Ara-A es fosforilado secuencialmente por quinasas para convertirse en el trifosfato de Ara-ATP. Esta es la forma activa del Ara-A y es a la vez un inhibidor y un sustrato de la ADN polimerasa viral. Cuando se utiliza como un sustrato para la ADN polimerasa viral, el Ara-ATP inhibe de forma competitiva el dATP que conduce a la formación de ADN 'defectuoso'. Aquí es donde Ara-ATP se incorpora en la cadena de ADN sustituyendo muchas de las bases de adenosina. Esto inhibe la síntesis de ADN, debido a que los puentes fosfodiéster deben ser más largos, desestabilizando la cadena (Yasuhara-Bell \& Lu 2010).

Adicionalmente, Perry et al. (1988), aislaron las micalamidas A y B de la esponja del género Mycale en Nueva Zelanda. La micalamida A inhibió la infección del VHS-1 a una concentración de $0,005 \mu \mathrm{g} \operatorname{disco}^{-1}$ y la micalamida B lo hizo con una dosis mínima de 0,001-0,002 $\mu \mathrm{g}$ disco $^{-1}$ (Perry et al.1990). El mecanismo de acción es mediante la inhibición de la síntesis de proteínas. Esta misma inhibición, también se describe a través de la unión de micalamida A al sitio E de la subunidad ribosómica de Haloarcula marismortui (Gurel et al. 2009).

Un año más tarde, se descubrió la ptilomicalina A, un alcaloide policíclico de guanidina aislado de la esponja del Caribe Ptilocaulis spiculifer, la cual demostró actividad contra el VHS-1 a una concentración de 0,2 $\mu \mathrm{g}$ $\mathrm{mL}^{-1}$ (Kashman et al. 1989).
Adicionalmente, el alcaloide bromoindólico, dragmacidina F, aislado de la esponja Halicortex sp. de la costa sur de Ustica (Italia) fue reportado como inhibidor del VHS-1 con una concentración efectiva $50\left(\mathrm{CE}_{50}\right)$ de 95,8 $\mu \mathrm{M}$ (Langlois et al. 1986, Cutignano et al. 2000). Otro ejemplo son los polisacáridos sulfatados de la esponja Celtodoryx girardae que inhiben el VHS-1 a concentraciones efectivas $\mathrm{CE}_{50}$ entre 5-33 $\mu \mathrm{g} \mathrm{mL} \mathrm{m}^{-1}$ (Rashid et al. 2009).

La 8-hidroximanzamina aislada de la esponja Pachypellina sp. proveniente de la playa de Manado en Sulawesi, Indonesia, mostró inhibición del VHS-2 con $\mathrm{CE}_{50}=0,05 \mu \mathrm{g} \mathrm{mL}^{-1}$ (Ichiba et al. 1994). La hamigerana B, uno de los 7 nuevos compuestos aislados de Hamigera tarangaensis, mostró un $100 \%$ de inhibición in vitro contra el VHS y el virus de la polio (Wellington et al. 2000).

Los alcaloides son un tipo de compuesto que ha despertado gran interés por su actividad contra el VHS. El alcaloide de 4-metilaptamina aislado de la esponja de Brasil Aaptos sp. inhibió la replicación de VHS-1 en células Vero de una manera dependiente de la dosis con un valor de $\mathrm{CE}_{50}$ de $2,4 \mu \mathrm{M}$, mostrando mayor potencia que el Aciclovir $(8,6 \mu \mathrm{M})$. La concentración requerida para inhibir la replicación de VHS-1 no era citotóxica, ya que el valor de $\mathrm{CE}_{50}$ de la 4-metilaptamina era igual a 72 $\mu \mathrm{M}$. Además se encontró que la 4-metilaptamina presenta actividad anti-VHS sostenida, incluso cuando se adiciona 
a las células Vero, 4 h después de la infección sugiriendo su actividad en los eventos iniciales de la replicación del VHS-1. Al parecer, el compuesto podría inhibir la expresión temprana de una proteína del VHS-1, la ICP27, que regula el corte, empalme, terminación, y exportación nuclear de los transcritos virales impidiendo así la replicación viral (Coutinho et al. 2002, Souza et al. 2007).

Recientemente se evaluó la actividad biológica de diferentes esponjas marinas recogidas en la costa de Brasil. Diversos extractos, tanto acuosos como orgánicos, fueron ensayados contra el VHS-1 por el método MTT utilizando células Vero, HEp-2 y MA104 (Da Silva et al. 2006). De acuerdo con los resultados, los extractos más prometedores son los extractos acuosos de Cliona sp., Agelas sp. y Tethya sp. con una $\mathrm{CE}_{50}$ de 136, 378 y $425 \mu \mathrm{g} \mathrm{mL} \mathrm{m}^{-1}$, respectivamente. Se postularon los siguientes mecanismos de acción: a) interacción con los receptores virales o celulares, inhibiendo la adsorción y penetración en las células; b) interacción y alteración de las moléculas de la envoltura viral, afectando la infectividad viral dependiente de envoltura (Da Silva et al. 2006).

\section{Virus de InMUNODEFICIENCIA Humana TIPO 1 (VIH-1)}

El aumento en la búsqueda y tamizaje de la actividad anti-VIH ha conducido al descubrimiento de numerosos compuestos (Tabla 2); sin embargo, los mecanismos de inhibición están todavía pobremente caracterizados (Fig. 1).

Los depsipéptidos contra el VIH, papuamidas, se aislaron de las esponjas Theonella mirabilis y Theonella swinhoeirecogidas a lo largo de la costa norte de Papúa

Tabla 2. Compuestos aislados de esponjas marinas con actividad anti-VIH / Compounds isolated from marine sponges with anti-HIV activity

\begin{tabular}{|c|c|c|c|}
\hline Especie & Sustancia & Clase & Referencia \\
\hline $\begin{array}{l}\text { Theonella mirabilis y } \\
\text { Theonella swinhoei }\end{array}$ & Papuamidas C y D & Depsipéptido & Ford et al. 1999 \\
\hline Xestospongia sp. & Haplosamatos A y B & Esteroles sulfatados & Qureshi \& Faulkner 1999 \\
\hline Disidea avara & Avarol & $\begin{array}{l}\text { Hidroquinona } \\
\text { sesquiterpeno }\end{array}$ & $\begin{array}{l}\text { Muller et al. 1985, 1987; } \\
\quad \text { Muller \& Schroder } 1991\end{array}$ \\
\hline Hippiospongia metachromia & Illimaquinona & Quinona & Loya \& Hizi 1993 \\
\hline Adocia sp. & Adociavirina & Proteína & O'Keefe et al. 1998 \\
\hline Halicortex sp. & Dragmacidina $\mathrm{F}$ & $\begin{array}{l}\text { Alcaloide } \\
\text { bromoindólico }\end{array}$ & Cutignano et al. 2000 \\
\hline Mixylla rosacea & Rosacelosa & $\begin{array}{r}\text { Polisacárido de glucosa } \\
\text { y sulfato de fucosa }\end{array}$ & Cimino et al. 2001 \\
\hline Clathria sp. & Clatesterol & Esterol sulfatado & Rudi et al. 2001 \\
\hline Sidonops microspinosa & Microspinosamida & Depsipéptido cíclico & Rashid et al. 2001 \\
\hline Petrosia sp. & Poliacetilenetriol & Depsipéptido & Loya et al. 2002 \\
\hline Homophymia sp. & Homopimina A & Ciclodepsipéptido & Zampella et al. 2008 \\
\hline Monanchora sp. & Crambescidina 826 & Alcaloide policíclico & Chang et al. 2003 \\
\hline Lendenfeldia & Dehidrofurodendina & Furanoterpeno & Chill et al. 2004 \\
\hline Neamphius huxleyi & Neampamida A & Depsipéptido & Oku et al. 2004 \\
\hline Petrosia similes & Petrosinas & $\begin{array}{l}\text { Alcaloides } \\
\text { bisquinolizidinos }\end{array}$ & Goud et al. 2003 \\
\hline $\begin{array}{l}\text { Theonella swinhoei y } \\
\text { Theonella cupola }\end{array}$ & Koshikamidas F y H & Péptidos no ribosomales & Plaza et al. 2010 \\
\hline Siliquariaspongia mirabilis & $\begin{array}{l}\text { Celebesida A y } \\
\text { Teopapuamida }\end{array}$ & Depsipéptidos & Plaza et al. 2009 \\
\hline Stelleta clavosa & Mirabamidas E, F, G y H & Depsipéptidos & Lu et al. 2011 \\
\hline Iotrochota baculifera & Baculiferinas & Alcaloides pirrolínicos & Fan et al. 2010 \\
\hline
\end{tabular}




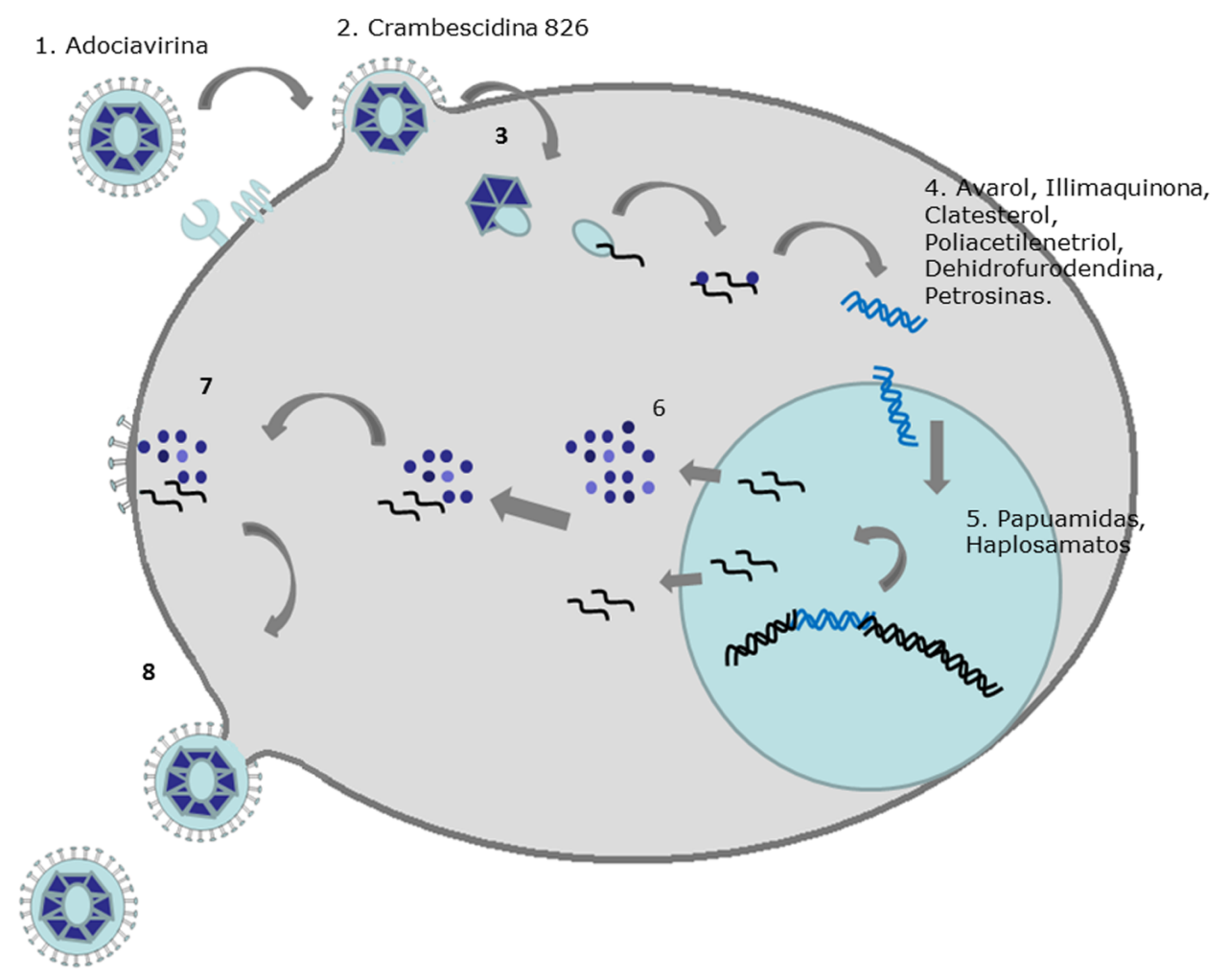

Figura 1. Ciclo de replicación del VIH-1 y los compuestos aislados de esponjas marinas con actividad anti-VIH-1. Se observa el ciclo de replicación del VIH-1 y los compuestos derivados de esponjas marinas que han presentado actividad antiviral en diferentes pasos del ciclo del virus. Unión [1], fusión [2], entrada [3], transcripción reversa [4], integración del ADN [5], producción de proteínas virales [6], empaquetamiento [7] y salida de nuevos viriones [8] / HIV replication cycle and compounds isolated from marine sponges with anti-HIV-1 activity. It is observe the HIV-1 replication cycle and the compounds derived from marine sponges that have shown antiviral activity in different steps of the viral cycle. Binding [1], fusion [2], entry [3], reverse transcription [4], DNA integration [5], viral protein production [6], assembly [7] and budding [8]

Nueva Guinea (Ford et al. 1999). Las papuamidas mostraron efectos antivirales potentes con una concentración efectiva de $3,6 \mathrm{ng} \mathrm{mL}^{-1}$ (Xie et al. 2008). El mecanismo de acción se debe a una interacción directa con la envoltura del virus (Andjelic et al. 2008) y la inhibición de la integrasa del virus con $\mathrm{Cl}_{50}$ de $50 \mu \mathrm{g} \mathrm{mL}^{-1}$. $\mathrm{Al}$ igual que los haplosamatos $\mathrm{A}$ y $\mathrm{B}$ que muestran una inhibición de la integrasa del VIH-1 con valores $\mathrm{CI}_{50}$ de 15 $\mu \mathrm{g} \mathrm{mL}{ }^{-1}$ (Qureshi \& Faulkner 1999).

Avarol es uno de los pocos compuestos para el cual los mecanismos de inhibición de la infección por VIH-1 son conocidos (Muller et al. 1985, 1987). Estudios in vitro y en animales indican que el avarol induce la respuesta inmune humoral, específicamente la producción de $\operatorname{IgG}$ e
IgM, e interfiere con los procesos post-transcripcionales de la infección viral. Adicionalmente, el avarol inhibe el VIH al bloquear la síntesis de los ARN de transferencia de Glutamina (ARNt) (Muller et al. 1985), lo cual es necesario para la síntesis de la proteasa viral. El avarol junto con metabolitos de esponjas como la avarona, los derivados de avarol, 6'-hidroxi Avarol y 3'-hidroxi Avarona, y varios derivados del fluoroglucinol (Muller $e t$ al. 1985, 1987), interactúan e inhiben la transcriptasa reversa (TR) viral (Loya et al. 2002); un efecto similar al presentado por la illimaquinona que inhibe la función de RNAsa H de la TR, inhibiendo así la replicación viral (Loya $\&$ Hizi 1993). 
De manera interesante, la ciclooxigenasa y la 5'lipoxigenasa son también inhibidas por el avarol, lo cual reduce los niveles de leucotrieno B4 y prostaglandina E2 en monocitos infectados in vitro con el VIH-1, induciendo un estado anti-inflamatorio, que disminuiría la disponibilidad de células activadas capaces de replicar el virus (Schröder et al. 1991); al mismo tiempo, el avarol reduce la expresión de genes en las células infectadas alterando la replicación viral (Belarbi \& Gómez 2003).

En 1998 se aisló en Nueva Zelanda, de la esponja Adocia sp., el compuesto adociavirina que mostró actividad anticitopática en células CEM-SS infectadas con el VIH-1. La adociavirina es activa contra diversas cepas y aislados del VIH-1 y 2, con valores de $\mathrm{CE}_{50}$ de $0,4 \mathrm{nM}$ a $400 \mathrm{nM}$. La potencia anti-VIH de adociavirina depende del tipo de célula hospedera, siendo los cultivos de macrófagos los más sensibles y los linfocitos de sangre periférica los más resistentes a la acción del compuesto (O’Keefe et al. 1998). La adociavirina se une tanto a los receptores celulares CD4 como a la glicoproteína de envoltura viral gp120 y probablemente mediante este mecanismo ejerce su actividad antiviral.

El alcaloide bromoindólico aislado de la esponja Halicortex sp. fue también reportado como inhibidor del VIH-1 con un $\mathrm{CE}_{50}$ de $0,91 \mu \mathrm{M}$. Este compuesto conocido como dragmacidina $\mathrm{F}$ retrasa la formación de sincitios por el VIH-1 (Cutignano et al. 2000).

En el 2001, a partir de un extracto acuoso de la esponja marina Mixylla rosacea, se aisló la rosacelosa, un nuevo compuesto polisacárido de glucosa y sulfato de fucosa con actividad anti-VIH-1. El compuesto inhibe la formación de sincitios después de la infección por el virus a una $\mathrm{CI}_{50}$ de $5 \mu \mathrm{g} \mathrm{mL}^{-1}$ en la línea celular MT-4 (Cimino et al. 2001). El clatesterol es un esterol sulfatado activo de la esponja del Mar Rojo Clathria sp., el cual ha demostrado ser activo contra la TR del VIH-1 a $10 \mu \mathrm{M}$ (Rudi et al. 2001). El depsipéptido cíclico inhibidor del VIH llamado microspinosamida, fue aislado de la esponja marina Sidonops microspinosa (Rashid et al. 2001). La microspinosamida inhibe el efecto citopático del VIH-1 con un valor de $\mathrm{CE}_{50}$ de $0,2 \mu \mathrm{g} \mathrm{mL} \mathrm{m}^{-1}$. El poliacetilenetriol aislado de la esponja marina Petrosia sp., mostró inhibición selectiva de la TR retroviral a una $\mathrm{CI}_{50}$ de $0,95 \mu \mathrm{M}$, en comparación con las $\mathrm{ADN}$ polimerasas celulares que las inhibe a una $\mathrm{CI}_{50}$ de $2,6 \mu \mathrm{M}$. Se ha postulado que realizando modificaciones estructurales de las cadenas laterales de la molécula plomo poliacetilénico se pueden producir nuevos medicamentos contra la infección por el VIH-1 que actúen de manera más potente (Loya et al. 2002).
Otro compuesto tipo depsipéptido que ha mostrado actividad anti-VIH es la homopimina A, un ciclodepsipéptido que se aisló de la esponja Homophymia sp. el cual mostró actividad inhibitoria del VIH-1 a concentraciones nanomolares en un ensayo basado en células XTT (Zampella et al. 2008).

El alcaloide policíclico tipo guanidina, la crambescidina 826 aislado de la esponja marina Monanchora sp. inhibe la fusión del VIH-1 in vitro a una $\mathrm{CI}_{50}$ de 1-3 $\mu \mathrm{M}$ (Chang et al. 2003). El furanoterpeno C22 aislado de la esponja de Madagascar Lendenfeldia, llamado dehidrofurodendina, se une directamente a la TR y bloquea las actividades ARN y ADN-dependientes de la ADN-polimerasa a una $\mathrm{CI}_{50}$ de 3,2-5,6 $\mu \mathrm{M}$ (Chill et al. 2004). Además, este compuesto tiene capacidad para inhibir la actividad de RNasa $\mathrm{H}$ de la TR del VIH-1 a una CI $_{50}$ de 29,5 mM (Chill et al. 2004).

Cabe destacar además la neampamida A, aislada en Papua Nueva Guinea de la esponja marina Neamphius huxleyi (Oku et al. 2004), la cual inhibe el efecto citopático del VIH-1 en ensayos in vitro a una $\mathrm{CE}_{50}$ de $28 \mathrm{nM}$. Además, se destacan 2 alcaloides bisquinolizidinos llamados petrosinas, que se aislaron de la esponja marina de la India Petrosia similes (Goud et al. 2003), las cuales inhiben la replicación del VIH-1 a una $\mathrm{CI}_{50}$ de 41,3-86,8 $\mu \mathrm{M}$ e inhibe la TR a una $\mathrm{CI}_{50}$ de 10,6-14,8 $\mu \mathrm{M}$ (Goud et al. 2003).

Las esponjas marinas del orden Lithistida (Theonellidae) son una fuente importante de péptidos no ribosomales que muestran propiedades anti-VIH, las koshikamidas $\mathrm{F}$ y $\mathrm{H}$ que fueron aislados de las esponjas T. swinhoei y la T. cupola (Plaza et al. 2010) inhiben la replicación del $\mathrm{VIH}$ a una $\mathrm{CI}_{50}$ de 2,3 y 5,5 $\mu \mathrm{M}$, respectivamente.

La celebesida A y la teopapuamida fueron aisladas de la esponja marina $S$. mirabilis y son activas contra el VIH-1 a una $\mathrm{CI}_{50}=0,002$ y $0,001 \mu \mathrm{M}$ respectivamente (Plaza et al. 2009). También se destacan los depsipéptidos denominados mirabamidas E, F, G y H que muestran una fuerte actividad contra el VIH-1 a una $\mathrm{CI}_{50}=121,62,68$ y $41 \mathrm{nM}$, respectivamente, los cuales fueron aislados de la esponja australiana Stelleta clavosa (Lu et al. 2011). Estos compuestos son estructuralmente similares a las mirabamidas A-C, pero con una sustitución del grupo treonina con su producto de deshidratación, el ácido 2aminobutenoico que se ha traducido en un aumento de casi el doble en la actividad de las mirabamidas E-H; dado que estas esponjas son filogenéticamente distintas, es posible que estos compuestos sean de origen microbiano asociado (Lu et al. 2011). 
En 2010, se aislaron 15 nuevos alcaloides pirrolínicos derivados de 3,4 dihidroxifenilalanina DOPA, con nombre baculiferinas A-O, las cuales fueron aisladas de la esponja marina China Iotrochota baculifera. Se encontró que 11 de ellas (Baculiferinas A, B, C, E, F, G, H, K, L, M y N) eran inhibidores potentes del VIH-1 tanto en células MAGI como en células MT-4 (Tabla 3) (Fan et al. 2010). Con el fin de dilucidar el mecanismo de acción, se evaluó la capacidad de unión de las baculiferinas con la gp41 recombinante (proteína transmembrana del VIH-1), la proteína Vif (factor de infectividad viral de VIH-1), y la APOBEC3G humana (un factor anti-viral intracelular innato) mediante una técnica que se denomina resonancia de plasmón superficial (SPR). La capacidad de unión de los alcaloides con la proteína diana se caracteriza por su respectivo valor $\mathrm{RU}$ (unidad de respuesta, $1 \mathrm{RU}=1 \mathrm{pg}$ $\mathrm{mm}^{-2}$ ), que se monitoriza en función del tiempo (Sensograma). En general, el valor RU hasta el rango de 100 se considera útil basado en los resultados de los bioensayos obtenidos en una biblioteca con 1.000 compuestos y con hierbas medicinales, mientras que la capacidad de unión con un máximo de 500 RU se considera que es una afinidad significativa. Las baculiferinas que contienen grupo $\mathrm{N}$ - ácido acético, como las L y M, tenían un potente afinidad de unión tanto con Vif (RU > 1800) como con APOBEC3G (RU > 2,170). Los alcaloides de tipo lactama I y J poseen una potente afinidad hacia gp41 $(\mathrm{RU}>1,350)$. Estos resultados sugieren que la capacidad de unión de los alcaloides con estas proteínas podría estar relacionado con su efecto antiviral (Fan et al. 2010).

Tabla 3. Actividad anti-VIH-1 de baculiferinas en células MT-4 y MAGI / Anti-HIV-1 activity of baculiferins in MT-4 and MAGI cells

\begin{tabular}{|c|c|c|}
\hline \multirow{2}{*}{ Baculiferina } & \multicolumn{2}{|c|}{ CI50 $\left(\mu \mathrm{gmL}^{-1}\right)$} \\
\hline & Células MT-4 & Células MAGI \\
\hline A & 7,6 & 3,7 \\
\hline B & 2,2 & 1,3 \\
\hline $\mathrm{C}$ & 8,4 & 1,2 \\
\hline E-F & 4,6 & 2,7 \\
\hline G & 3,2 & 4,4 \\
\hline $\mathrm{H}$ & 1,4 & 1,3 \\
\hline $\mathrm{K}$ & 5,5 & $<0,4$ \\
\hline $\mathrm{L}$ & 7,0 & 4,1 \\
\hline M & 5,0 & 0,2 \\
\hline $\mathrm{N}$ & 4,4 & $<0,1$ \\
\hline
\end{tabular}

\section{ViRUS DE LA INFLUENZA}

Un objetivo en la lucha contra el virus de la influenza es la búsqueda de nuevos inhibidores de la neuraminidasa viral. Recientemente, se aislaron 3 compuestos sulfatados activos denominados caliceramidas A, B y C de la esponja marina Discodermia caliz (Nakao et al. 2001); estos compuestos inhiben la neuraminidasa de la bacteria Clostridium perfringens con valores de $\mathrm{CI}_{50}$ de $0,4,0,2$ y $0,8 \mu \mathrm{g} \mathrm{mL} \mathrm{mL}^{-1}$, respectivamente; dichos valores eran ligeramente más potentes que el ácido 4-acetil neuramínico $\mathrm{CI}_{50}=1,5 \mathrm{mg} \mathrm{mL}^{-1}$.

Por otra parte, en el 2002 se aisló y purificó el nobilosido, una penasterol saponina, de la esponja marina E. nobilis, la cual se recogió de la isla de Shikine-jima en Japón y reveló la presencia de un grupo carboxílico en C30, además de restos de ácidos urónicos. El nobilosido inhibe la neuraminidasa de la bacteria Clostridium perfrigens con una $\mathrm{CI}_{50}$ de $0,5 \mu \mathrm{M}$ (Takada et al. 2002).

En el 2004 se aislaron las schulzeinas A, B y C, 3 trisulfatos benzo[á]quinolizidínicos, de la esponja marina Penares schulzei, que exhiben actividad inhibidora de la neuraminidasa de la bacteria Clostridium perfrigens con una $\mathrm{CI}_{50}=60 \mathrm{mM}$ (Yang et al. 2011). Todos estos compuestos inhibidores de la neuraminidasa tienen potencial de inhibir el virus de la influenza.

En el 2012 se aislaron 2 compuestos de un hongo asociado a la esponja Callyspongia sp. colectado en Sanya, China. Los compuestos llamados iso-D8646-2-6 y D8646-2-6 presentaron actividad contra el virus de la influenza A (H1N1) en un ensayo de inhibición de efecto citopático (ECP); mostrando valores inhibidores de 91,5 y 101,3 $\mu \mathrm{M}$ para el iso-D8646-2-6 y D8646-2-6 respectivamente (Peng et al. 2012).

\section{Coronavirus}

El etil-ester del esculetin-4-acido carboxílico aislado de la esponja marina brasileña Axinella corrugata es activo contra el coronavirus causante del SARS, inhibiendo la proteasa $3 \mathrm{CL}$ con una $\mathrm{CI}_{50}=46 \mu \mathrm{M}$, entre otras (Mayer et al. 2011). Este es un hallazgo potencialmente significativo porque la proteasa 3CL es un 'objetivo de alto perfil' en el desarrollo de fármacos contra SARS, ya que parece estar implicada en la liberación de proteínas víricas replicativas (Mayer et al. 2011).

\section{COMPUESTOS CON ACTIVIDAD POLIVIRAL}

Dentro de este grupo se destacan la hamigerana B y los weinberesteroles. La hamigerana B aislada de Hamigera tarangaensis muestra una inhibición in vitro del $100 \%$ 
de los virus del herpes y de la polio (Wellington et al. 2000), mientras que los Weinberesteroles A y B aislados de Petrosia weinbergi muestra actividad contra los virus de la leucemia felina, la influenza y el corona virus (Sun et al.1991, Muller \& Schroder 1991).

\section{Conclusiones}

El arduo trabajo en la investigación durante los últimos 60 años ha conducido al aislamiento e identificación de varios compuestos bioactivos de uso terapéutico potencial en el tratamiento antiviral. Por esta razón, es esencial que los gobiernos y los laboratorios farmacéuticos se esfuercen para incluir organismos marinos en sus programas de descubrimiento y desarrollo de fármacos, especialmente para enfermedades infecciosas recurrentes, cuyo agente etiológico tenga gran capacidad de mutación. En ese esfuerzo se debe incluir la infección por el VIH, virus para el cual aunque existen tratamientos disponibles, se presenta una alta resistencia viral, debida a las mutaciones virales o se presentan efectos secundarios que dificultan la adherencia al tratamiento y deterioran la calidad de vida del individuo infectado.

Se puede afirmar que los compuestos naturales con actividad antiviral para los que se conoce el punto del ciclo replicativo en el que tienen actividad y el mecanismo de acción son pocos. Esto se debe a la dificultad que representa el cultivo de muchos virus, y la dificultad técnica para poder evaluar, en forma independiente, cada etapa del ciclo de replicación, lo cual ha dificultado el desarrollo de nuevos medicamentos antivirales.

A pesar de los enormes avances en investigación para desarrollar medicamentos antivirales potentes y más cuando la mayoría de los tratamientos actualmente disponibles provienen precisamente de compuestos naturales, existen limitantes que podrían afectar dicho desarrollo; el hecho de que no existan pruebas de laboratorio estándar a nivel mundial que permitan una determinación más homogénea de la actividad antiviral de diferentes compuestos, dificulta la aplicabilidad de los resultados; además, existen laboratorios que no cumplen con los estándares de infraestructura necesarios para controlar virus peligrosos, a pesar de tener los compuestos naturales disponibles. En contraste, en la actualidad las nuevas tecnologías permiten el estudio de la expresión de genes la cual es útil para identificar moléculas blanco de la actividad biológica de los compuestos, mejorando sustancialmente el descubrimiento de nuevas terapias.
Finalmente, es importante resaltar que una gran cantidad de productos naturales de origen marino con un futuro prometedor como principios activos en medicamentos no podrían llegar a convertirse en potenciales agentes terapéuticos debido a problemas de suministro; por esta razón se debe explorar el desarrollo de estrategias de síntesis química o de fermentación adecuadas para garantizar su disponibilidad en el futuro.

\section{Agradecimientos}

Este trabajo fue financiado por el Comité para el Desarrollo de la Investigación, CODI de la Universidad de Antioquia, Proyecto 01591 y el Programa de Sostenibilidad del Grupo Inmunovirología 2013-2014.

\section{LITERATURA CITADA}

Aiello A, M D'Esposito, E Fattorusso, M Menna \& W Müller. 2006. Novel bioactive bromopyrrole alkaloids from the Mediterranean sponge Axinella verrucosa. Bioorganic \& Medicinal Chemistry 14(1): 17-24.

Andjelic C, V Planelles \& L Barrows. 2008. Characterizing the Anti-HIV Activity of Papuamide A. Marine Drugs 6: 528-549.

Aswell JF, GP Allen, AT Jamieson, DE Campbell \& GA Gentry. 1977. Antiviral activity of arabinosylthyrnine in herpesviral replication: mechanism of action in vivo and in vitro. Antimicrobial Agents and Chemotherapy 12: 243254.

Belarbi E \& C Gómez. 2003. Producing drugs from marine sponges. Biotechnology Advances 21: 585-598.

Bergmann W \& DC Burke. 1955. Contributions to the study of marine products. XXXIX. The nucleosides of sponges. III. Spongothymidine and spongouridine. The Journal of Organic Chemistry 20: 1501-1507.

Bergmann W \& RJ Feeney. 1950. The isolation of a new thymine pentoside from sponges. Journal of the American Chemical Society 72: 2809-2810.

Bergmann W \& RJ Feeney. 1951. Contributions to the study of marine products, XXXII: the nucleosides of sponges, I. The Journal of Organic Chemistry 16(6): 981-987.

Bickmeyer U, C Drechsler, M Köck \& M Assmann. 2004. Brominated pyrrole alkaloids from marine Agelas sponges reduce depolarization-induced cellular calcium elevation. Toxicon 44(1): 45-51.

Buchanan MS, AR Carroll, GA Fechner, A Boyle, M Simpson, R Addepalli, VM Avery, JNA Hooper, T Cheung, H Chen \& RJ Quinn. 2008. Aplysamine 6, an alkaloidal inhibitor of isoprenylcysteine carboxyl methyltransferase from the sponge Pseudoceratina sp. Journal of Natural Products 71(6): 1066-1067. 
Bula-Meyer G. 1989. Micro y macroalgas marinas alelopáticas: Biología, toxinas y significado ecofisiológico. Revista Ingeniería Pesquera 9(1/2): 110.

Chang L, NF Whittaker \& CA Bewley. 2003. Crambesidin 826 and dehyrocrambine A: a new polycyclic guanidine alkaloids from the marine sponge Monanchora sp. that inhibit HIV-1 fusion. Journal of Natural Products 66: 14901494.

Chill L, A Rudi, M Aknin, S Loya, A Hizi \& Y Kashman. 2004. New sesterterpenes from Madagascan Lendenfeldia sponges. Tetrahedron 60: 10619-10626.

Cimino P, G Bifulco, A Casapullo, I Bruno, L GomezPaloma \& R Riccio. 2001. Isolation and NMR characterization of rosacelose, a novel sulfated polysaccharide from the sponge Mixylla rosacea. Carbohydrate Research 334(1): 39-47.

Compagnone R, R Avila, A Suarez, O Abrams, H Rangel \& F Arvelo. 1999. 11-Deoxyfistularin-3, a new cytotoxic metabolite from the Caribbean sponge Aplysinafistularis insularis. Journal of Natural Products 62: 1443-1444.

Corredor JE, CR Wilkinson, VP Vicente, JM Morrel \& E Otero. 1988. Nitrate release by Caribbeean reef sponges. Limnology and Oceanography 33(1): 114-120.

Coutinho AF, B Chanas, TMLE Souza, ICPPA Frugrulhetti \& R Epifanio. 2002. Anti HSV-1 alkaloids from a feeding deterrent marine sponge of the genus Aaptos. Heterocycles 57: $1265-1272$.

Cutignano A, G Bifulco, I Bruno, A Casapullo, L GomezPaloma \& R Riccio. 2000. Dragmacidin F: A new antiviral bromoindole alkaloid from the Mediterranean sponge Halicortex sp. Tetrahedron 56(23): 3743-3748.

Da Silva AC, JM Kratz, FM Farias, AT Henriques, J Dos Santos, RM Leonel, C Lerner, B Mothes, CR Barardi \& CM Simões. 2006. In vitro antiviral activity of marine sponges collected off Brazilian coast. Biological \& Pharmaceutical Bulletin 29: 135-140.

Fan G, Z Li, S Shen, Y Zeng, Y Yang, M Xu, T Bruhn, H Bruhn, J Morschhäuser, G Bringmann \& W Lin. 2010. Baculiferins A-O, O-sulfated pyrrole alkaloids with antiHIV-1 activity, from the Chinese marine sponge Iotrochota baculifera. Bioorganic \& Medicinal Chemistry 18(15): 54665474.

Faulkner DJ. 2002. Marine natural products. Journal of Natural Products 19: 1-48.

Ford PW, KR Gustafson, TC McKee, N Shigematsu, LK Maurizi, LK Pannell, DE Williams, ED De Silva, $P$ Lassota, TM Alien, R Van Soest, RJ Anderson \& MR Boyd. 1999. Papuamides A-D, HIV-inhibitory and cytotoxic depsipeptides form the sponges Theonella mirabilis and Theonella swinhoei collected in Papua New Guinea. Journal of the American Chemical Society 121: 5899-5909.
Galeano E \& A Martínez. 2007. Antimicrobial activity of marine sponges from Urabá Gulf, Colombian Caribbean region. Journal de Mycologie Médicale 17(1): 21-24.

Gerwick WH \& BS Moore. 2012. Lessons from the past and charting the future of marine nature products drug discovery and chemical biology. Chemistry \& Biology 19(1): 85-98.

Goud TV, NS Reddy, NR Swamy, TS Ram \& Y Venkateswarlu. 2003. Anti-HIV active petrosins from the marine sponge Petrosia similes. Biological \& Pharmaceutical Bulletin 51: 990-993.

Gul W \& M Hamann. 2005. Indole alkaloid marine natural products: An established source of cancer drug leads with considerable promise for the control of parasitic, neurological and other diseases. Life Sciences 78(5): 442-453.

Gurel G, G Blaha, T Steitz \& P Moore. 2009. The structures of Triacetyloleandomycin and Mycalamide A bound to the large ribosomal subunit of Haloarcula marismortui. Antimicrobial Agents and Chemotherapy 53: 5010-5014.

Ichiba T, J Corgiat, P Scheuer \& M Kelly-Borges. 1994. 8Hydroxymanzamine A, a beta-carboline alkaloid from a sponge, Pachypellina sp. Journal of Natural Products 57: 168-170.

Kashman Y, S Hirsh, OJ McConnell, I Ohtani, T Kusumi \& H Kakisawa. 1989. Ptilomycalin A: a novel polycyclic guanidine alkaloid of marine origin. Journal of the American Chemical Society 111(24): 8925-8926.

Kong F \& RJ Andersen. 1996. Polymastiamides B-F, novel steroid/amino acid conjugates isolated from the Norwegian marine sponge Polymastia boletiformis. Journal of Natural Products 59(4): 379-385.

Langlois M, JP Allard, F Nugier \& M Aymard. 1986. A rapid and automated colorimetric assay for evaluating the sensitivity of herpes simplex strains to antiviral drugs. Journal of Biological Standardization 14(3): 201-211.

Loya S \& A Hizi. 1993. The interaction of illimaquinone, a selective inhibitor of the RNase $\mathrm{H}$ activity, with the reverse transcriptases of human immunodeficiency and murine leukemia retroviruses. Journal of Biological Chemistry 268: 9323-9328.

Loya S, A Rudi, Y Kashman \& A Hizi. 2002. Mode of inhibition of HIV-1 reverse transcriptase by polyacetylenetriol, a novel inhibitor of RNA- and DNA-directed DNA polymerases. Biochemical Journal 362: 685-692.

Lu Z, RM Van Wagoner, MK Harper, HL Baker, JNA Hooper \& CA Bewley. 2011. Mirabamides E-H, HIV-inhibitory depsipeptides from the sponge Stelletta clavosa. Journal of Natural Products 74: 185-193.

Márquez DM, E Galeano \& A Martínez. 2003. Productos naturales con actividad antimicrobiana. Parte I. Vitae 10(2): 61-71. 
Márquez DM, E Galeano \& A Martínez. 2004. Productos naturales con actividad antimicrobiana. Parte II. Vitae 11(1): $35-41$

Mayer A, AD Rodríguez, RGS Berlinck \& N Fusetani. 2011. Marine pharmacology in 2007-8: Marine compounds with antibacterial, anticoagulant, antifungal, antiinflammatory, antimalarial, antiprotozoal, antituberculosis, and antiviral activities; affecting the immune and nervous system, and other miscellaneous mechanisms of action. Comparative Biochemistry and Physiology Part C: Toxicology \& Pharmacology 153(2): 191-222.

Mayer AMS, KB Glaser, C Cuevas, RS Jacobs, W Kem, RD Little, JM McIntosh, DJ Newman, BC Potts \& DE Shuster. 2010. The odyssey of marine pharmaceuticals: a current pipeline perspective. Trends in Pharmacological Sciences 31: 255-265.

Miller RL, JP Iltis \& F Rapp. 1977. Differential effect of arabino furanosyl thymine of the replication of human herpes viruses. Journal of Virology 23: 679-684.

Muller WEG \& HC Schroder. 1991. Cell biological aspects of HIV-1 infection: effects of the anti-HIV-1 agent avarol. International Journal of Sports Medicine 12: S43-S49.

Muller WEG, A Maidhof, RK Zahn, HC Schroder, MJ Gasic, D Heidemann, A Bernd, B Kurelec, E Eich \& HC Seibert. 1985. Influence of the antileukemic activity of the novel cytostatic agent avarone and its analogues in vitro and in vivo. Cancer Research 45: 4822-4826.

Muller WEG, C Sobel, B Diehl-Seifert, A Maidhof \& HC Schroder. 1987. Influence of the antileukemic and antihuman immunodeficiency virus agent avarol on selected immune responses in vitro and in vivo. Biochemical Pharmacology 36: 1489-1494.

Murakamia N, M Kawanishia, H Mostaqul, J Li, S Itagaki, T Horiib \& M Kobayashi. 2003. New antimalarial peroxides with in vivo potency derived from spongean metabolites. Bioorganic \& Medicinal Chemistry Letters 13(22): 4081-4084.

Nakao Y, K Takada, S Matsunaga \& N Fusetani. 2001. Calyceramides A-C: neuraminidase inhibitory sulfated ceramides from the marine sponge Discodermia calyx. Tetrahedron 57: 3013-3017.

Narsinh L \& E Werner. 2004. Biotechnological potential of marine sponges. Current Science 86(11): 1506-1512.

O'Keefe BR, T Erim, JA Beutler, JH Cardellina II, RJ Gulakowski, BL Krepps, JB McMahon, RC Sowder II, DG Johnson, RW Buckheit Jr, S Halliday \& MR Boyd. 1998. Isolation and characterization of adociavirin, a novel HIV-inhibitory protein from the sponge Adocia sp. Federation of European Biochemical Societies Letters 431(1): 85-90.
Oku N, KR Gustafson, LK Cartner, JA Wilson, N Shigematsu, S Hess, LK Pannell, MR Boyd \& JB McMahon. 2004. Neamphamide A, a new HIV-inhibitory depsipeptide from the Papua New Guinea marine sponge Neamphius huxleyi. Journal of Natural Products 67: 14071411.

Peng J, J Jiao, J Li, W Wang, Q Gu, T Zhu \& D Li. 2012. Pyronepolyene C-glucosides with NF-jB inhibitory and anti-influenza A viral (H1N1) activities from the spongeassociated fungus Epicoccum sp. JJY40. Bioorganic \& Medicinal Chemistry Letters 22: 3188-3190.

Pérez-López P, E Ternonb, S González-García, G GentaJouveb, G Feijooa, OP Thomas \& MT Moreira. 2014. Environmental solutions for the sustainable production of bioactive natural products from the marine sponge Crambe crambe. Science of the Total Environment 475: 71-82.

Perry N, J Blunt, M Munro \& L Pannell. 1988. Mycalamide A, an antiviral compound from a New Zealand sponge of the genus Mycale. Journal of the American Chemical Society 110: 4850-4851.

Perry NB, JW Blunt, MHG Munro \& AM Thompson. 1990. Antiviral and antitumor agents from a New Zealand sponge, Mycale sp. 2. Structures and solution conformations of mycalamides A and B. The Journal of Organic Chemistry 55: 223-227.

Piñol M \& J Palazón. 1993. Fisiología y bioquímica vegetal, 582 pp. McGraw-Hill, Madrid.

Plaza A, G Bifulco, JL Keffer, JR Lloyd, HL Baker \& CA Bewley. 2009. Celebesides A-C and theopapuamides B-D, depsipeptides from an Indonesian sponge that inhibit HIV1 entry. The Journal of Organic Chemistry 74: 504-512.

Plaza A, G Bifulco, M Masullo, JR Lloyd, JL Keffer \& PL Colin. 2010. Mutremdamide A and koshikamides $\mathrm{C}-\mathrm{H}$, peptide inhibitors of HIV-1 entry from different Thennella species. The Journal of Organic Chemistry 75: 4344-4355.

Privat de Garilhe M. 1964. Effect of 2 arbinose nucleosides on the multiplication of herpes virus and vaccine in cell culture. Comptes Rendus Hebdomadaires des Séances de l'Académie des Sciences 259: 2725-2728.

Qureshi A \& DJ Faulkner. 1999. Haplosamates A and B: new steroidal sulfamate esters from two haploclerid sponges. Tetrahedron 55: 8323-8330.

Rashid MA, KR Gustafson, LK Cartner, N Shigematsu, LK Pannell \& MR Boyd. 2001. Microspinosamide, a new HIV-inhibitory cyclic depsipeptide from the marine sponge Sidonops microspinosa. Journal of Natural Products 64: 117-121.

Rashid ZM, E Lahaye \& D Defer. 2009. Isolation of a sulphated polysaccharide from a recently discovered sponge species (Celtodoryxgirardae) and determination of its antiherpetic activity. International Journal of Biological Macromolecules 44(3): 286-293. 
Rudi A, T Yosief, S Loya, A Hizi, M Schleyer \& Y Kashman. 2001. Clathsterol, a novel anti-HIV-1 RT sulfated sterol from the sponge Clathria species. Journal of Natural Products 64: 1451-1453.

Rützler K \& C Feller. 1987. Mangrove swamp communities. Oceanus 30(4): 16-24.

Schröder H, M Bégin, R Klöcking, E Matthes, A Sarma, M Gašiæ \& W Müller. 1991. Avarol restores the altered prostaglandin and leukotriene metabolism in monocytes infected with human immunodeficiency virus type 1 . Virus Research 21: 213-223.

Schwartz PM, C Shipman Jr \& JC Drach. 1976. Antiviral activity of arabinosyladenine and arabinosylhypoxanthine in herpes simplex virus infected KB cells: selective inhibition of viral deoxyribonucleic acid synthesis in the presence of an adenosine deaminase inhibitor. Antimicrobial Agents and Chemotherapy 10: 64-74.

Sipkema D, MC Franssen, R Osinga, J Tramper \& RH Wijffels. 2005. Marine sponges as pharmacy. Marine Biotechnology 7: 142-162.

Soares DC, CG Pereira, MAA Meireles \& EM Saraiva. 2007. Leishmanicidal activity of a supercritical fluid fraction obtained from Tabernaemontana catharinensis. Parasitology International 56(2): 135-139

Souza T, J Abrantes, $R$ de A Epifanio, C Fontes \& I Frugulhetti. 2007. The Alkaloid 4-Methylaaptamine isolated from the sponge Aaptos aaptos impairs herpes simplex virus type 1 penetration and immediate-early protein synthesis. Planta Medica 73: 200-205.

Sun HH, SS Cross, M Gunasekera \& FE Koehn. 1991. Weinbersteroldisulfates A and B, antiviral steroid sulfates from the sponge Petrosia weinbergi. Tetrahedron 47: 11851190.

Takada K, Y Nakao, S Matsunaga, RWM van Soest \& N Fusetani. 2002. Nobiloside, a new neuraminidase inhibitory triterpenoidal saponin from the marine sponge Erylus nobilis. Journal of Natural Products 65: 411-413.
Tziveleka LA, C Vagias \& V Roussis. 2003. Natural products with anti-HIV activity from marine organisms. Current Topics in Medicinal Chemistry 3: 1512-1535.

Utagawa T, H Morisawa, T Miyoshi, F Yoshinaga, A Yamazaki \& K Mitsugi. 1980. A novel and simple method for the preparation of adenine arabinoside by bacterial transglycosylation reaction. Federation of European Biochemical Societies Letters 109: 261-263.

Wellington KD, RC Cambie, PS Rutledge \& PR Bergquist. 2000. Chemistry of sponges, 19: novel bioactive metabolites from Hamigera tarangaensis. Journal of Natural Products 63: 79-85.

Xie W, D Ding, W Zi, G Li \& D Ma. 2008. Total synthesis and structure assignment of papuamide $\mathrm{B}$, a potent marine cyclodepsipeptide with anti-HIV properties. Angewandte Chemie International Edition 47: 2844-2848.

Yang C, Y Bao, P Liang, J Ye, Ai-E Wang \& P Huang. 2011 A new approach to the $\mathrm{C} 28$ fatty acid chain of the marine natural products schulzeines $\mathrm{B}$ and $\mathrm{C}$ : a concise diastereoselective total synthesis of(-)-schulzeine B. Tetrahedron 67(34): 6281-6288.

Yasuhara-Bell J \& Y Lu. 2010. Marine compounds and their antiviral activities. Review Article. Antiviral Research 86(3): 231-240.

Zampella A, V Sepe, P Luciano, F Bellotta, MC Monti \& MV D'Auria. 2008. Homophymine A, an anti-HIV cyclodepsipeptide from the sponge Homophymia sp. The Journal of Organic Chemistry 73: 5319-5327.

Zea SE. 1998. Estado actual del conocimiento en sistemática de esponjas marinas (Porifera) del Caribe colombiano. Boletín Ecotrópica: Ecosistemas Tropicales 33: 43-59.

Zhang W, S Xuea, Q Zhaoa, X Zhanga, M Jina, , X Yua \& Q Yuana. 2003. Biopotentials of marine sponges from China oceans: past and future. Biomolecular Engineering 20(4-6): 413-419. 